\title{
Achieving consistent and equitable access to post mastectomy breast reconstruction
}

\author{
Anne C. O’Neill \\ Division of Plastic Surgery, Department of Surgery and Surgical Oncology, University Health Network, University of Toronto, Toronto, Canada \\ Correspondence to: Anne C. O’Neill. 8N-867 200 Elizabeth Street Toronto, Ontario M5G 2C4, Canada. Email: Anne.O’Neill@uhn.ca. \\ Provenance and Peer Review: This article was commissioned by the editorial office, Gland Surgery. The article did not undergo external peer review. \\ Comment on: Jeevan R. Reconstructive utilisation and outcomes following mastectomy surgery in women with breast cancer treated in England. Ann \\ R Coll Surg Engl 2020;102:110-4.
}

Submitted Jan 20, 2020. Accepted for publication Feb 11, 2020.

doi: $10.21037 /$ gs.2020.02.13

View this article at: http://dx.doi.org/10.21037/gs.2020.02.13

One in eight women will be diagnosed with breast cancer in their lifetime and for many the recommended treatment plan will include mastectomy. For many women this can result in loss of femininity, sexual identity and long-term psychosocial impairment. With increasing numbers of women surviving the disease, minimizing the impact of cancer and its treatment has become an important element of the care pathway. Breast reconstruction has been shown to mitigate the adverse effects of mastectomy and improve health related quality of life for breast cancer survivors (1). Although both immediate and delayed reconstruction can be effective, women who undergo reconstruction at the time of mastectomy can be protected from a period of significant psychosocial distress and diminished sexual well-being (2). However, in spite of these proven benefits post mastectomy breast reconstruction (PMBR) is not consistently offered as part of the breast cancer treatment plan.

In their recent publication in the Annals of the Royal College of Surgeons in England Ranjeet Jeevan and colleagues summarize an extensive body of work that examines the current use of PMBR in England (3). In a series of studies, the authors provide a comprehensive overview of mastectomy and reconstruction rates based on data from the National Hospital Episode Statistics (HES) database and a prospective national audit (4-8). They report immediate breast reconstruction (IBR) rates in the region of $20 \%$ for women undergoing mastectomy in English cancer network hospitals. While there are a number of positive findings such as a steady increase in IBR rates over time and high levels of patient satisfaction with care, the studies identify significant inequity in the utilisation of IBR across centres. Older women, non-Caucasians and those from economically deprived areas were found to have significantly decreased IBR rates.

The work of Jeevan et al. makes an important contribution to the global literature in this field. While there has been significant research interest in the rates of PMBR, the majority of studies come from the United States (US). The rates of IBR in the US do not necessarily reflect those seen internationally and so there is a need for more data from different healthcare models (9-11). American studies have long recognized lower rates of PMBR in nonCaucasian women and those from lower socioeconomic groups (12). They have led the field in implementing strategies that aim to improve access to PMBR. The discrepancies in PMBR rates in the US are often attributed to the inherent inequities in healthcare coverage. Studies demonstrate that while legislation such as the Womens Health and Cancer Rights Act [1998] and the New York State Breast Cancer Provider Discussion Law [2010] have improved rates of IBR among underserved women, significant discrepancies persist $(13,14)$.

Jeevan's work illustrates that even in a National Health Service where all citizens are entitled to free healthcare, there are significant racial and socioeconomic disparities in uptake of reconstructive surgery among women with breast cancer with regional rates ranging from $9-43 \%$. This echoes the findings of a previous Canadian study that reported wide variations in PMBR rates in a Universal Healthcare single-payer system (15). These studies indicate 
that discrepancies in utilisation of PMBR are not simply due to the inability to pay for the surgery itself. Women from lower income groups are less likely to have supplementary insurance or employment benefits to cover the additional indirect costs of more complex surgery with extended recovery times. A study examining IBR rates in Department of Defense personnel is one of the few that found no difference between white and black women, suggesting that when women have an equal-access healthcare system combined with a similar level of workplace support, racial disparity can be overcome (16).

Perhaps the most interesting aspect of Jeevan et al.'s work is the exploration of the number of women who received an offer of IBR. They report that less than $50 \%$ of women are offered IBR with offer rates ranging from $24-75 \%$. Although the National Health Service in England has guidelines in place mandating that IBR should be discussed and offered to all patients unless there is a contraindication, this wide variation in offer rates suggests that this is not uniformly applied. Surprisingly they report no correlation between the offer rate and the IBR rate which is contrary to other studies that found the most common reason patients did not have IBR was that it was not proposed by their surgeon (9).

The studies report that offers were less likely in older women, those with comorbidities, higher tumor burden and the anticipated need for adjuvant radiotherapy.

The studies do not provide any qualitative analysis of how the offer is made. There is a stark difference between providing patients with unbiased information on the risks and benefits of IBR and simply asking them if they want a reconstruction. For the majority of women who have been diagnosed with breast cancer their priority is to have surgical removal of the tumour as soon as possible and they are less likely to pursue IBR if they feel further consultations may delay this process. There are currently no standardised guidelines for how an offer of IBR should be made. In most cases the surgeon who decides that the patient is unsuitable is the surgical oncologist and not the reconstructive surgeon. Patients who do not avail of IBR often later report that they did not receive sufficient information. Ideally all newly diagnosed breast cancer patients should have the opportunity to meet with a plastic surgeon who can provide a specialised opinion on the risks and benefits of IBR in their individual case. This would ensure that patients are provided with adequate information to actively participate in the decision-making process and prevent long-term decision regret.
The studies suggest that, in most cases an IBR offer is withheld because the surgeon did not consider the patient a suitable candidate. This subjective rather than evidence-based decision making may be strongly influenced by surgeon bias. There was significant discrimination against older women in spite of a large body of evidence demonstrating that PMBR is safe and effective in this population (17). Jeevan et al. suggest that surgeons are adhering to current guidelines in England, except with respect to age. However, these guidelines allow for significant subjectivity as they state that IBR should be offered unless there is a clinical contraindication. With advances in surgical techniques there are relatively few absolute contraindications to IBR. While some patients are undoubtedly at increased risk for post-operative complications, in many cases these will be minor and effective breast reconstruction can still be safely achieved. These short-term complications must be balanced against the long-term health related quality of life benefits that IBR can offer. There is a need for more high-quality studies to explore the true relationship between perceived risk factors and clinical outcomes so that evidence-based guidelines can be developed. We need to move away from generalised contraindications towards a more personalised approach where the clinical profile as well as the needs and wishes of an individual patient can be considered, so that the safest and most effective breast reconstruction option can be offered. Validated clinical risk assessment tools can aid preoperative patient counseling and facilitate more objective patient selection for IBR $(18,19)$.

Though the work of Jeevan et al. demonstrates a positive trajectory, IBR rates in the UK and other countries fall far behind those reported in some other studies. While there is no optimal target rate for IBR, this suggests that there is scope for further improvement. A concerted ongoing effort will be required to overcome multifactorial barriers to the routine and equitable integration of IBR into the breast cancer care pathway if rates are to be increased in the future. Patient education is an essential step in this process. Women must be provided with impartial and accurate information on the risks and benefits of breast reconstruction and empowered with an awareness of the options available to them. This information must be delivered in a culturally sensitive and language appropriate manner and be made available to women of all socioeconomic groups and education levels. This information can be overwhelming, especially in the setting of a recent cancer diagnosis and patients will require significant support in the decision- 
making process. Decision aids can help women to make the most effective choices in a time sensitive manner, ensuring that the opportunity for long-term gains in health-related quality of life is not lost (20).

Although Jeevan's studies suggest that service or resource issues did not significantly affect the decision to offer IBR the marked regional disparities suggest this may not be the case. Furthermore, IBR rates were shown to be higher in the small number of private institutions that participated. Studies have demonstrated that the financial status of a hospital significantly affects rates of IBR $(21,22)$. Patients are more likely to be offered IBR at teaching hospitals in metropolitan areas with higher numbers of plastic surgeons (23). There is an inverse relationship between the geographical distance to a centre that offers reconstruction and the likelihood of IBR (24). If IBR is to be universally accessible there must be appropriate and uniform infrastructure to support this. Centres that provide mastectomy but do not have reconstructive services should be affiliated to a reconstructive centre and have a standardised protocol for accelerated referral of cases to ensure patients can have IBR without delaying their cancer treatment.

While barriers to IBR are undoubtedly multi-factorial, Jeevan et al.'s work suggests that the attitudes of surgeons remain a key area to be addressed. Breast reconstruction must be accepted as an integral part of the cancer care pathway based on strong evidence that it can improve longterm psychosexual well-being. Reconstructive surgeons must work closely with other members of the multidisciplinary cancer team to ensure that IBR can be offered in an efficient and effective manner without compromising other essential elements of cancer care. Surgical oncologists face many challenges when trying to meet cancer treatment targets and it is understandable that in the face of often limited resources breast reconstruction may not be prioritised. However, we can no longer accept such blatant disparities in the quality of breast cancer care. The National Accreditation Program for Breast Centres in the US endorses a standard of care where surgical oncologists offer all patients a referral to a plastic surgeon at the time of cancer diagnosis. Making this standard practice would help ensure that all women who wish to avail of IBR may have the opportunity to do so irrespective of age, race and socioeconomic status (25).

Jeevan et al. are to be congratulated on this significant undertaking that provides important insights into the provision of IBR in England and indeed globally. Studies such as these are essential if we are to understand and address the factors that prevent women from accessing the benefits of breast reconstruction.

\section{Acknowledgments}

Funding: None.

\section{Footnote}

Conflicts of Interest: The author has completed the ICMJE uniform disclosure form (available at http://dx.doi. org/10.21037/gs.2020.02.13). O'Neill AC has no conflicts of interest to declare.

Ethical Statement: The author is accountable for all aspects of the work in ensuring that questions related to the accuracy or integrity of any part of the work are appropriately investigated and resolved.

Open Access Statement: This is an Open Access article distributed in accordance with the Creative Commons Attribution-NonCommercial-NoDerivs 4.0 International License (CC BY-NC-ND 4.0), which permits the noncommercial replication and distribution of the article with the strict proviso that no changes or edits are made and the original work is properly cited (including links to both the formal publication through the relevant DOI and the license). See: https://creativecommons.org/licenses/by-nc-nd/4.0/.

\section{References}

1. Zhong T, McCarthy C, Min S, et al. Patient satisfaction and health-related quality of life after autologous tissue breast reconstruction: a prospective analysis of early postoperative outcomes. Cancer 2012;118:1701-9.

2. Zhong T, Hu J, Bagher S, et al. A Comparison of Psychological Response, Body Image, Sexuality, and Quality of Life between Immediate and Delayed Autologous Tissue Breast Reconstruction: A Prospective Long-Term Outcome Study. Plast Reconstr Surg 2016;138:772-80.

3. Jeevan R. Reconstructive utilisation and outcomes following mastectomy surgery in women with breast cancer treated in England. Ann R Coll Surg Engl 2020;102:110-4.

4. Jeevan R, Cromwell DA, Browne JP, et al. Regional variation in use of immediate breast reconstruction after mastectomy for breast cancer in England. Eur J Surg 
Oncol 2010;36:750-5.

5. Jeevan R, Cromwell DA, Browne JP, et al. Findings of a national comparative audit of mastectomy and breast reconstruction surgery in England. J Plast Reconstr Aesthet Surg 2014;67:1333-44.

6. Jeevan R, Browne JP, Pereira J, et al. Socioeconomic deprivation and inpatient complication rates following mastectomy and breast reconstruction surgery. Br J Surg 2015;102:1064-70.

7. Jeevan R, Browne JP, Gulliver-Clarke C, et al. Association between age and access to immediate breast reconstruction in women undergoing mastectomy for breast cancer. Br J Surg 2017;104:555-61.

8. Browne JP, Jeevan R, Gulliver-Clarke C, et al. The association between complications and quality of life after mastectomy and breast reconstruction for breast cancer. Cancer 2017;123:3460-7.

9. Naoura I, Mazouni C, Ghanimeh J, et al. Factors influencing the decision to offer immediate breast reconstruction after mastectomy for ductal carcinoma in situ (DCIS): the Institut Gustave Roussy Breast Cancer Study Group experience. Breast 2013;22:673-5.

10. Kamali P, van Bommel A, Becherer B, et al. Immediate Breast Reconstruction in The Netherlands and the United States: A Proof-of-Concept to Internationally Compare Quality of Care Using Cancer Registry Data. Plast Reconstr Surg 2019;144:565e-74e.

11. Feng Y, Flitcroft K, van Leeuwen MT, et al. Patterns of immediate breast reconstruction in New South Wales, Australia: a population-based study. ANZ J Surg 2019;89:1230-5.

12. Epstein S, Tran BN, Cohen JB, et al. Racial disparities in postmastectomy breast reconstruction: National trends in utilization from 2005 to 2014. Cancer 2018;124:2774-84.

13. Yang RL, Newman AS, Lin IC, et al. Trends in immediate breast reconstruction across insurance groups after enactment of breast cancer legislation. Cancer 2013;119:2462-8.

14. Fu RH, Baser O, Li L, et al. The Effect of the Breast Cancer Provider Discussion Law on Breast Reconstruction Rates in New York State. Plast Reconstr Surg 2019;144:560-8.

15. Zhong T, Fernandes KA, Saskin R, et al. Barriers to

Cite this article as: O'Neill AC. Achieving consistent and equitable access to post mastectomy breast reconstruction. Gland Surg 2020;9(4):1082-1085. doi: 10.21037/gs.2020.02.13 immediate breast reconstruction in the Canadian universal health care system. J Clin Oncol 2014;32:2133-41.

16. Enewold LR, McGlynn KA, Zahm SH, et al. Breast reconstruction after mastectomy among Department of Defense beneficiaries by race. Cancer 2014;120:3033-9.

17. Song D, Slater K, Papsdorf M, et al. Autologous Breast Reconstruction in Women Older Than 65 Years Versus Women Younger Than 65 Years: A Multi-Center Analysis. Ann Plast Surg 2016;76:155-63.

18. Roy M, Sebastiampillai S, Haykal S, et al. Development and validation of a risk stratification model for immediate microvascular breast reconstruction. J Surg Oncol 2019;120:1177-83.

19. Roy M, Sebastiampillai S, Zhong T, et al. Synergistic Interaction Increases Complication Rates following Microvascular Breast Reconstruction. Plast Reconstr Surg 2019;144:1e-8e.

20. Metcalfe K, Zhong T, O'Neill AC, et al. Development and testing of a decision aid for women considering delayed breast reconstruction. J Plast Reconstr Aesthet Surg 2018;71:318-26.

21. Offodile AC 2nd, Muldoon LD, Gani F, et al. The site of care matters: An examination of the relationship between high Medicaid burden hospitals and the use, cost, and complications of immediate breast reconstruction after mastectomy. Cancer 2018;124:346-55.

22. Richards CA, Rundle AG, Wright JD, et al. Association Between Hospital Financial Distress and Immediate Breast Reconstruction Surgery After Mastectomy Among Women With Ductal Carcinoma In Situ. JAMA Surg 2018;153:344-51.

23. Retrouvey H, Solaja O, Gagliardi AR, et al. Barriers of Access to Breast Reconstruction: A Systematic Review. Plast Reconstr Surg 2019;143:465e-76e.

24. Platt J, Zhong T, Moineddin R, et al. Geographic Variation Immediate and Delayed Breast Reconstruction Utilization in Ontario, Canada and Plastic Surgeon Availability: A Population-Based Observational Study. World J Surg 2015;39:1909-21.

25. Mahmoudi E, Lu Y, Metz AK, et al. Association of a Policy Mandating Physician-Patient Communication With Racial/Ethnic Disparities in Postmastectomy Breast Reconstruction. JAMA Surg 2017;152:775-83. 